\title{
Performance of Fractioned and Nonfractioned Equalizers with High-Level QAM
}

\author{
RAMÓN AGUSTÍ, MEMBER, IEEE, FERNANDO CASADEVALL, AND JUAN J. OLMOS
}

\begin{abstract}
This paper presents a comparative study of 16-QAM, 32QAM, 64-QAM, and 128-QAM $140 \mathrm{Mbit} / \mathrm{s}$ digital radio in the presence of multipath fading. Conventional $T$-spaced and fractional tapspaced equalizers have been considered to compensate for the ISI-induced symbols. The performance measure used is the bit error ratio (BER); thus, upper and lower bounds on BER have been developed. Based on this calculation procedure, results showing the net fade margin versus the gross fade margin have been obtained in a variety of cases that include the presence of both nonlinear and linear equalizer structures.
\end{abstract}

\section{INTRODUCTION}

T $\mathrm{HE}$ crowded conditions prevailing in many regions of the radio spectrum combined with the increased emphasis on digital transmission have created a need for improved spectrum utilization techniques. In this context, high-level modulation schemes such as $M$-QAM ( $M=$ $32,64,128$, etc. ) have been proposed in conjunction with the already classical 16 QAM [1]. On the other hand, however, multipath propagation appearing in the digital radio systems causes an increasing degradation as the number of levels increases in the aforementioned modulations. An adequate parameter to assess its performance in the presence of propagation distortion is obtained by means of the "net fade margin"' (NFM), defined as [2]

$$
\text { Prob. }(A \geq \mathrm{NFM})=\text { Prob. }\left(\mathrm{BER} \geq 10^{-\gamma}\right)
$$

where $A$ is the fading depth, BER is the system bit error ratio, and $\gamma=3$ or 6 , according to CCIR recommendations. Classical numerical methods based on the previously computed moments of the random variable, accounting for ISI and ICI, have been carried out successfully. These methods, however, are cumbersome and become impractical when the number of levels of the constellation $M$-QAM is high, as happens when 32 or more levels are considered in the modulation. In order to overcome these difficulties, we have developed tight bounds on the BER based on a modification of the Milewski bounds [3] proposed for PAM systems. Once an adequate tool to obtain the BER is available, two baseband equalization techniques are analyzed: $T$-spaced equalizers (TSE's) and fractional tap-spaced equalizers (FTSE's). Finally, we present a comparative study of the $M$-QAM

\footnotetext{
Manuscript received July 28, 1986; revised December 12, 1986.

The authors are with the Department of Communications, Escuela Técnica Superior de Ingenieros de Telecomunicación, Apdo. 30.002, 08071 Barcelona, Spain.

IEEE Log Number 8613834
}

modulations $(M=16,32,64,128)$ in the presence of linear and nonlinear baseband equalizers.

\section{Transmission Model}

Fig. 1 shows the low-pass equivalent model of the transmission system. The transmitted signal can be formulated as

$$
\begin{aligned}
S(t)= & \sum_{k=-\infty}^{\infty}\left(a_{k}+j b_{k}\right) \delta(t-k T) \\
= & \sum_{k=-\infty}^{\infty} d_{k} \delta(t-k T)
\end{aligned}
$$

where $\left\{a_{k}\right\},\left\{b_{k}\right\}$ are data sequences of duration $T$ for the in-phase and quadrature channels. They are $\pm 1, \pm 3$, $\cdots, \pm(2 L-1)$ with $L=2$ for 16 QAM, $L=3$ for 32 QAM, $L=4$ for 64 QAM, and $L=6$ for 128 QAM. Moreover, $a_{k}$ and $a_{l}$ (respectively, $b_{k}$ and $b_{l}$ ) are independent random variables $\forall k \neq l$. However, $a_{k}$ and $b_{k}$ are independent in 16 QAM and 64 QAM, but dependent in 32 QAM and 128 QAM.

The overall filtering transfer function $H_{T}(f) \cdot H_{R}(f)$ is of the raised-cosine type with a chosen rolloff factor equal to 0.5 . The filtering is split equally between the transmitter and the receiver. $H_{c}(f)$ introduces the presence of selective fading in the radio link. This transfer funtion is formulated by means of the Rummler model [4].

$$
H_{c}(f)=a\left[1-b e^{ \pm j 2 \pi\left(f-f_{d}\right) \tau}\right]
$$

where $a$ and $b$ control the scale and shape of the fade, respectively, $A=-20 \log _{10}(1-b)$ is the fading depth; $\tau=6.3 \mathrm{~ns}, f_{d}$ is the notch offset frequency, and the plus and minus signs in the exponent correspond to nonminimum-phase and minimum-phase fading, respectively. $\bar{\varphi}$ is introduced to model the real behavior of a carrier recovery loop. We have considered a Costas loop as representative [5]. $n(t)$ is a Gaussian complex noise with phase $\left(n_{x}(t)\right)$ and quadrature $\left(n_{y}(t)\right)$ components uncorrelated.

The received signal $r(t)$ can be expressed by

$$
\begin{aligned}
r(t)= & \sum_{k=-\infty}^{\infty}\left[a_{k} P(t-k T)-b_{k} Q(t-k T)\right] \\
& +j \sum_{k=-\infty}^{\infty}\left[b_{k} P(t-k T)+a_{k} Q(t-k T)\right] \\
& +n_{x}(t)+j n_{y}(t)
\end{aligned}
$$




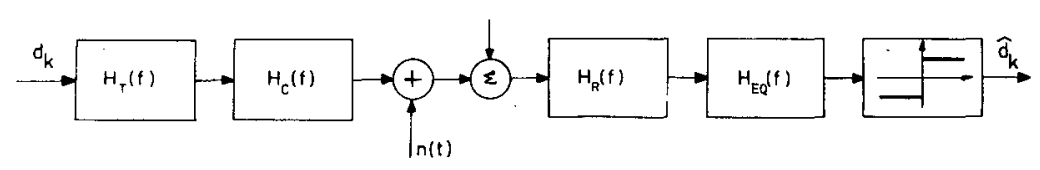

Fig. 1. Low-pass equivalent model of the transmission system.

where, in general

$$
\begin{aligned}
P(t)+j Q(t) & =\left[F ^ { - 1 } \left\{H_{T}(f)\right.\right. \\
& \left.\left.\cdot H_{c}(f) \cdot H_{R}(f) \cdot H_{\mathrm{EQ}}(f) \cdot e^{-j \bar{\varphi}}\right\}\right] \cdot G,
\end{aligned}
$$

$H_{\mathrm{EQ}}(f)$ is the equalizer transfer function and $G$ is a gain factor introduced to be considered the presence of automatic gain control (AGC). The sampling instant $t_{0}$ has been chosen according to the classical square law envelope timing recovery [6].

Four baseband equalizer structures are analyzed:

1) a linear equalizer with baud period $T$ spacing;

2) a linear equalizer with spacing $T^{\prime}=T / 2$;

3) a nonlinear equalizer with baud period spacing on feedforward and feedback filters

4) a nonlinear equalizer with fractional spacing $T^{\prime}=$ $T / 2$ on the forward filter and baud period spacing on the feedback filter.

In all cases, the minimum mean-square error (MMSE) technique has been adopted to calculate the tap values.

\section{Bit Error Probability Calculation}

In order to perform a comparative study of the different equalizer structures for high-level QAM modulations using the bit error probability as a quality parameter, we present the following bounds.

\section{A. 16-QAM Bounds on BER}

Following [3], we arrange the absolute values of the interfering samples ranging in decreasing order of magnitude. In our case, however, we arrange indistinctly both ISI and ICI. So we have $\left|S_{1}\right| \geq\left|S_{2}\right| \geq \cdots \geq\left|S_{i}\right| \geq$ $\cdots$ where $S_{i}$ is either $P\left(t_{0}+k T\right) / P\left(t_{0}\right)$ or $Q\left(t_{0}+\right.$ $k T) / P\left(t_{0}\right)$, according to its absolute value.

We define

$$
\xi=\beta_{1}\left|S_{1}\right|+\sum_{k=2}^{\infty} \beta_{k}\left|S_{k}\right|=\beta_{1}\left|S_{1}\right|+x_{2}
$$

where $\beta_{k} \epsilon( \pm 1, \pm 3)$ and

$$
x_{2}=\sum_{k=2}^{\infty} \beta_{k}\left|S_{k}\right| \text {. }
$$

Consequently, the $P_{16 \mathrm{QAM}}(\epsilon)$ can be formulated after some algebraic effort as

$$
\begin{aligned}
P_{16 \mathrm{QAM}}= & \frac{3}{8}\left[\frac { 1 } { 4 } E _ { x _ { 2 } } \left\{\operatorname{erfc}\left[\left(1-3\left|S_{1}\right|+x_{2}\right) \rho_{0}\right]\right.\right. \\
& \left.\left.\cdot \sum_{m=1}^{4} \frac{\operatorname{erfc}\left[\left(1-e_{1, m}+x_{2}\right) \rho_{0}\right]}{\operatorname{erfc}\left[\left(1-3\left|S_{1}\right|+x_{2}\right) \rho_{0}\right]}\right\}\right]
\end{aligned}
$$

where $E_{x_{2}}(\cdot)$ denotes the mean of the random variable $x_{2}$, $\rho_{0}=\sqrt{\mathrm{SNR} / 10}, e_{1}=3\left|S_{1}\right|$, and $e_{1, m}=\beta_{1}\left|S_{1}\right|$ with $\beta_{1} \in( \pm 1, \pm 3)$.

Taking into account that for $\Delta V>0$, erfc $(V+$ $\Delta V) / \operatorname{erfc}(V)$ is a monotonous decreasing function, the expression (6) can be upper bounded by

$$
\begin{aligned}
& P_{16 \mathrm{QAM}}(\epsilon) \leq \underbrace{\frac{3}{8} E_{x_{2}}\left\{\operatorname{erfc}\left[\left(1-3\left|S_{1}\right|+x_{2}\right) \rho_{0}\right]\right\}}_{\cdots a "} \\
& \cdot \sum_{m=1}^{4} \frac{1}{4} \frac{\operatorname{erfc}\left[\left(1-3\left|S_{1}\right|+\min x_{2}-e_{1, m}+e_{1}\right) \rho_{0}\right]}{\operatorname{erfc}\left[\left(1-3\left|S_{1}\right|+\min x_{2}\right) \rho_{0}\right]}
\end{aligned}
$$

where we have defined

$$
\min x_{2}=-3 \sum_{k=2}^{\infty}\left|S_{k}\right| \text {. }
$$

By repeating the above process $l$ times with the term " $a$," we finally have in the limit $(l \rightarrow \infty)$ that

$$
\begin{aligned}
P_{16 \mathrm{QAM}} \leq & \frac{3}{8} \operatorname{erfc}\left[\left(1-D_{p}\right) \rho_{0}\right] \prod_{j=1}^{\infty} \\
& \cdot \frac{1}{4}\left\{\sum_{m=1}^{4} \frac{\operatorname{erfc}\left[\left(1-D_{p}+e_{j}-e_{j, m}\right) \rho_{0}\right]}{\operatorname{erfc}\left[\left(1-D_{p}\right) \rho_{0}\right]}\right\}
\end{aligned}
$$

where we have considered

$$
\min x_{j+1}=-3 \sum_{i=j+1}^{\infty}\left|S_{i}\right|=-\sum_{i=j+1}^{\infty} e_{i}
$$

and have introduced the peak distortion

$$
D_{p}=3 \sum_{n=1}^{\infty}\left|S_{n}\right| \text {. }
$$

Analogously, a lower bound on $P_{16 \mathrm{QAM}}(\epsilon)$ can be found if we consider that

$$
\frac{\operatorname{erfc}(V+\Delta V)}{\operatorname{erfc}(V)} \geq \frac{\operatorname{erfc}(W+\Delta W)}{\operatorname{erfc}(W)}
$$

for $W>V$ and $\Delta>0$. In that case,

$$
\begin{aligned}
P_{16 \mathrm{QAM}}(\epsilon) \geq \frac{3}{8} \operatorname{erfc}\left[\left(1-D_{p}\right) \rho_{0}\right] \prod_{j=1}^{\infty} \\
\cdot \frac{1}{4}\left\{\sum_{m=1}^{4} \frac{\operatorname{erfc}\left[\left(1+D_{p}-2 E_{j-1}-e_{j}-e_{j, m}\right) \rho_{0}\right]}{\operatorname{erfc}\left[\left(1+D_{p}-2 E_{j}\right) \rho_{0}\right]}\right\}
\end{aligned}
$$


where

$$
E_{j}=\sum_{n=1}^{j} 3\left|S_{n}\right|
$$

In both upper and lower bounds, the first term is the error probability we would have obtained if we had bounded the interference by the eye diagram aperture. The second term includes the correction factor necessary to consider the random nature of the transmitted digital message. Due to the ordering in absolute value of the interfering samples, the convergence of this second term is fast. So only six or seven terms are required to attain a good precision in all analyzed cases.

\section{B. 32-QAM Bounds on BER}

The calculation of bounds on BER in a 32 QAM can be performed in a way similar to that in the 16-QAM case. At first, an ordering in absolute value of the terms $P\left(t_{0}\right.$ $+n T) / P\left(t_{0}\right)$ and $Q\left(t_{0}+n T\right) / P\left(t_{0}\right)$, denoted as $\left|S_{n}\right|$, is to be made. Then we form

$$
\xi=\beta_{1}\left|S_{1}\right|+\sum_{n=2}^{\infty} \beta_{n}\left|S_{n}\right|=\beta_{1}\left|S_{1}\right|+\dot{x}_{2}
$$

where $\beta_{n} \epsilon( \pm 1, \pm 3, \pm 5)$ and

$$
x_{2}=\sum_{n=2}^{\infty} \beta_{n}\left|S_{n}\right| \text {. }
$$

Now, however, the 32 possible levels of the random variable $\beta_{\dot{n}}$ are distributed as shown in Table $I$.

The 32-QAM BER can be formulated as

$$
\begin{aligned}
P_{32 \mathrm{QAM}}(\epsilon)= & \frac{7}{24} E_{x_{2}}\left\{\operatorname{erfc}\left[\left(1-5\left|S_{1}\right|+x_{2}\right) \rho_{0}\right]\right. \\
& \cdot\left[\sum_{m=1}^{4} \frac{3}{16} \frac{\operatorname{erfc}\left[\left(1-f_{1, m}+x_{2}\right) \rho_{0}\right]}{\operatorname{erfc}\left[\left(1-5\left|S_{1}\right|+x_{2}\right) \mid \rho_{0}\right]}\right. \\
& \left.\left.+\sum_{k=1}^{2} \frac{2}{16} \frac{\operatorname{erfc}\left[\left(1-f_{1, k}+x_{2}\right) \rho_{0}\right]}{\operatorname{erfc}\left[\left(1-5\left|S_{1}\right|+x_{2}\right) \rho_{0}\right]}\right]\right\}
\end{aligned}
$$

with

$$
\begin{aligned}
f_{1, m} & =\beta_{1}\left|S_{1}\right| \text { and } \beta_{1} \epsilon( \pm 1, \pm 3) \\
f_{1, k} & =\beta_{1}\left|S_{1}\right| \text { and } \beta_{1} \epsilon( \pm 5) \\
\rho_{0} & =\sqrt{\mathrm{SNR} / 20} .
\end{aligned}
$$

By denoting $e_{1}=5\left|S_{1}\right|$ and $e_{1, m}=\beta_{1}\left|S_{1}\right|$ with $\beta_{1} \epsilon( \pm 1$, $\pm 3, \pm 5$ ), the above formula can be bounded as

$P_{32 \mathrm{QAM}} \leq \frac{7}{24} E_{x_{2}}\left\{\operatorname{erfc}\left[\left(1-5\left|S_{1}\right|+x_{2}\right) \rho_{0}\right]\right\}$

$\cdot\left[\sum_{m=1}^{6} \frac{3}{16} \frac{\operatorname{erfc}\left[\left(1-5\left|S_{1}\right|+\min x_{2}-e_{1, m}+e_{1}\right) \rho_{0}\right]}{\operatorname{erfc}\left[\left(1-5\left|S_{1}\right|+\min x_{2}\right) \rho_{0}\right]}-\sum_{k=1}^{2} \frac{1}{16} \frac{\operatorname{erfc}\left[\left(1-5\left|S_{1}\right|+\min x_{2}-e_{1, k}+e_{1}\right) \rho_{0}\right]}{\operatorname{erfc}\left[\left(1-5\left|S_{1}\right|+\min x_{2}\right) \rho_{0}\right]}\right]$

\begin{tabular}{ccccccc}
\hline$\beta_{n}$ & -5 & -3 & -1 & 1 & 3 & 5 \\
\hline Prob. $\left(\beta_{n}\right)$ & $4 / 32$ & $6 / 32$ & $6 / 32$ & $6 / 32$ & $6 / 32$ & $4 / 32$ \\
\hline
\end{tabular}

where $\min x_{2}=-5 \Sigma_{n=2}^{\infty}\left|S_{n}\right|$ and $e_{1, k}=\beta_{1}\left|S_{1}\right|$ with $\beta_{1} \epsilon( \pm 5)$. By repeating iteratively $l$ times the same process with the first term, we obtain

$$
\begin{aligned}
& P_{32 \mathrm{QAM}}(\epsilon) \leq \frac{7}{24} \operatorname{erfc}\left\{\left(1-D_{p}\right) \rho_{0}\right\} \\
& \prod_{j=1}^{\infty}\left[\sum_{m=1}^{6} \frac{3}{16} \frac{\operatorname{erfc}\left[\left(1-D_{p}+e_{j}-e_{j, m}\right) \rho_{0}\right]}{\operatorname{erfc}\left[\left(1-D_{p}\right) \rho_{0}\right]}\right. \\
&\left.-\sum_{k=1}^{2} \frac{1}{16} \frac{\operatorname{erfc}\left[\left(1-D_{p}+e_{j}-e_{j, k}\right) \rho_{0}\right]}{\operatorname{erfc}\left[\left(1-D_{p}\right) \rho_{0}\right]}\right]
\end{aligned}
$$

where $D_{p}=5 \Sigma_{n=1}^{\infty}\left|S_{n}\right|$ is the peak distortion. Analogously, for the lower bound we have

$$
\begin{aligned}
P_{32 \mathrm{QAM}}(\epsilon) \geq & \frac{7}{24} \operatorname{erfc}\left[\left(1-D_{p}\right) \rho_{0}\right] \\
\prod_{j=1}^{\infty} & {\left[\sum_{m=1}^{6} \frac{3}{16} \frac{\operatorname{erfc}\left[\left(1+D_{p}-2 E_{j-1}-e_{j}-e_{j, m}\right) \rho_{0}\right]}{\operatorname{erfc}\left[\left(1+D_{p}-2 E_{j}\right) \rho_{0}\right]}\right.} \\
& \left.-\sum_{k=1}^{2} \frac{1}{16} \frac{\operatorname{erfc}\left[\left(1+D_{p}-2 E_{j-1}-e_{j}-e_{j, k}\right) \rho_{0}\right]}{\operatorname{erfc}\left[\left(1+D_{p}-2 E_{j}\right) \rho_{0}\right]}\right]
\end{aligned}
$$

where $E_{j}=5 \Sigma_{n=1}^{j}\left|S_{n}\right|$. Again, as we see in the 16-QAM case, the first term corresponds to the eye diagram aperture bound, the second term being a correction factor that converges with $j=6$ or 7 .

\section{64- and 128-QAM Bounds on BER}

The calculation of the 64- and 128-QAM bounds is similar to the calculation of the 16- and 32-QAM bounds, respectively. So only the final expressions are shown, as follows: 
64 QAM:

$$
\begin{aligned}
& \frac{7}{32} \operatorname{erfc}(D) \prod_{j=1}^{\infty} \frac{1}{8} \sum_{m=1}^{\infty} F_{1}(j, m) \\
& \quad \leq P_{64 \mathrm{QAM}}(\epsilon) \leq \frac{7}{32} \operatorname{erfc}(\dot{D}) \prod_{j=1}^{\infty} \frac{1}{8} \sum_{m=1}^{8} F_{2}(j, m)
\end{aligned}
$$

where

$$
\begin{aligned}
D & =\left(1-D_{p}\right) \rho_{0} \\
D_{p} & =7 \sum_{n=1}^{\infty}\left|S_{n}\right|, \rho_{0}=\sqrt{\mathrm{SNR} / 42}, \\
F_{1}(j, m) & =\frac{\operatorname{erfc}\left[\left(1-D_{p}+e_{j}-e_{j, m}\right) \rho_{0}\right]}{\operatorname{erfc}\left[\left(1-D_{p}\right) \rho_{0}\right]}, \\
F_{2}(j, m) & =\frac{\operatorname{erfc}\left[\left(1+D_{p}-2 E_{j-1}-e_{j}-e_{j, m}\right) \rho_{0}\right]}{\operatorname{erfc}\left[\left(1+D_{p}-2 E_{j}\right) \rho_{0}\right]}, \\
e_{j} & =7\left|S_{j}\right|, e_{j, m}=\beta_{j}\left|S_{j}\right| ; \beta_{j} \epsilon( \pm 1, \pm 3, \pm 5, \pm 7)
\end{aligned}
$$

and

$$
E_{j}=\sum_{n=1}^{j} 7\left|S_{n}\right|
$$

\section{QAM:}

$$
\begin{aligned}
P_{128 \mathrm{QAM}}(\epsilon) \leq & \frac{15}{96} \operatorname{erfc}(D) \prod_{j=1}^{\infty}\left[\frac{6}{64} \sum_{m=1}^{12} F_{1}(j, m)\right. \\
& \left.-\frac{2}{64} \sum_{k=1}^{4} F_{1}(j, k)\right] \\
P_{128 \mathrm{QAM}}(\epsilon) \geq & \frac{15}{96} \operatorname{erfc}(D) \prod_{j=1}^{\infty}\left[\frac{6}{64} \sum_{m=1}^{12} F_{2}(j, m)\right. \\
& \left.-\frac{2}{64} \sum_{k=1}^{4} F_{2}(j, k)\right]
\end{aligned}
$$

where $D, F_{1}(j, m)$ and $F_{2}(j, m)$ are shown above and

$$
\begin{gathered}
D_{p}=11 \sum_{n=1}^{\infty}\left|S_{n}\right|, e_{j}=11\left|S_{j}\right|, e_{j, k}=\beta_{j}\left|S_{j}\right| \\
\text { for } \quad \beta_{j} \epsilon( \pm 9, \pm 11), \\
e_{j, m}=\beta_{j}\left|S_{j}\right| \quad \text { for } \quad \beta_{j} \epsilon( \pm 1, \pm 3, \pm 5, \pm 7, \pm 9 ; \pm 11), \\
E_{j}=\sum_{n=1}^{j} 11\left|S_{n}\right|, \quad \text { and } \quad \rho_{0}=\sqrt{\mathrm{SNR} / 82} .
\end{gathered}
$$

\section{Results And Discussion}

In order to illustrate the behavior of the proposed bounds, two generic cases are shown in Fig. 2 for 32 QAM and 64 QAM, respectively. In these examples, as in the rest of the analyzed cases, a truncated impulse response of $10 T$ duration has been considered. Besides the upper and lower bound on the BER, Fig. 2 also shows the

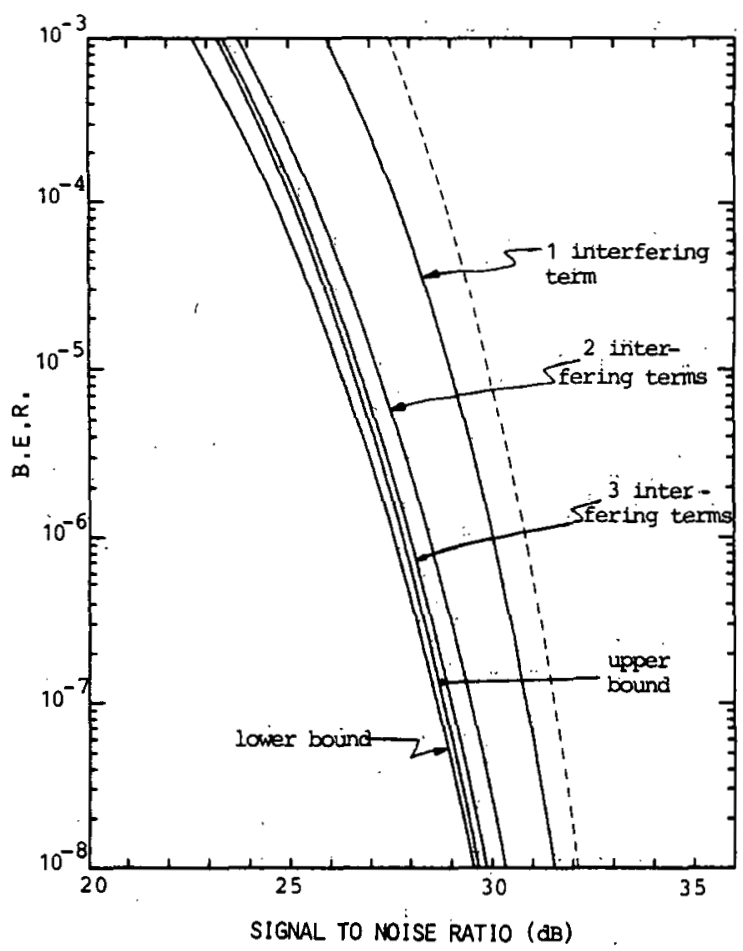

(a).

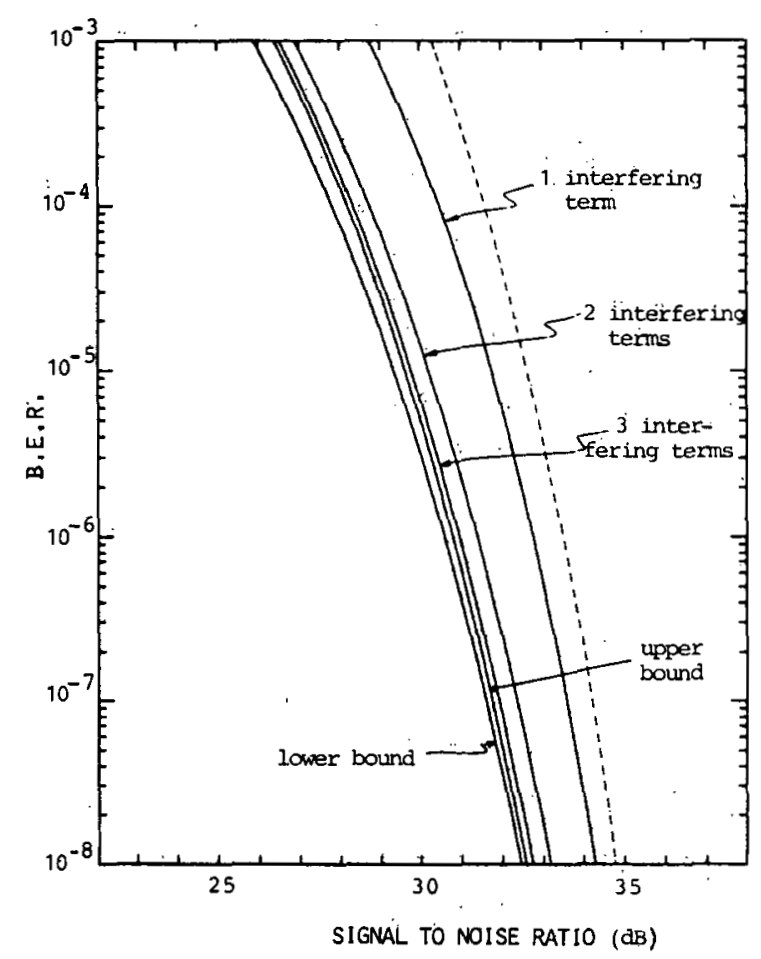

(b)

Fig. 2. BER bounds versus SNR. Dashed line: BER bound using the maximal eye diagram opening. (a) 32-QAM system. (b) 64-QAM system.

convergence rate as the number of interfering terms increases. Since only seven terms are necessary to ensure a good convergence, the procedure is really fast. Moreover, the bounds are very tight; that is, at most $0.5 \mathrm{~dB}$ error in the SNR can be guaranteed.

We have obtained results for a $2^{n}$-QAM system $(n \doteq$ $4,5,6,7)$ and the channel parameters $\tau / T=0.2205$ and 

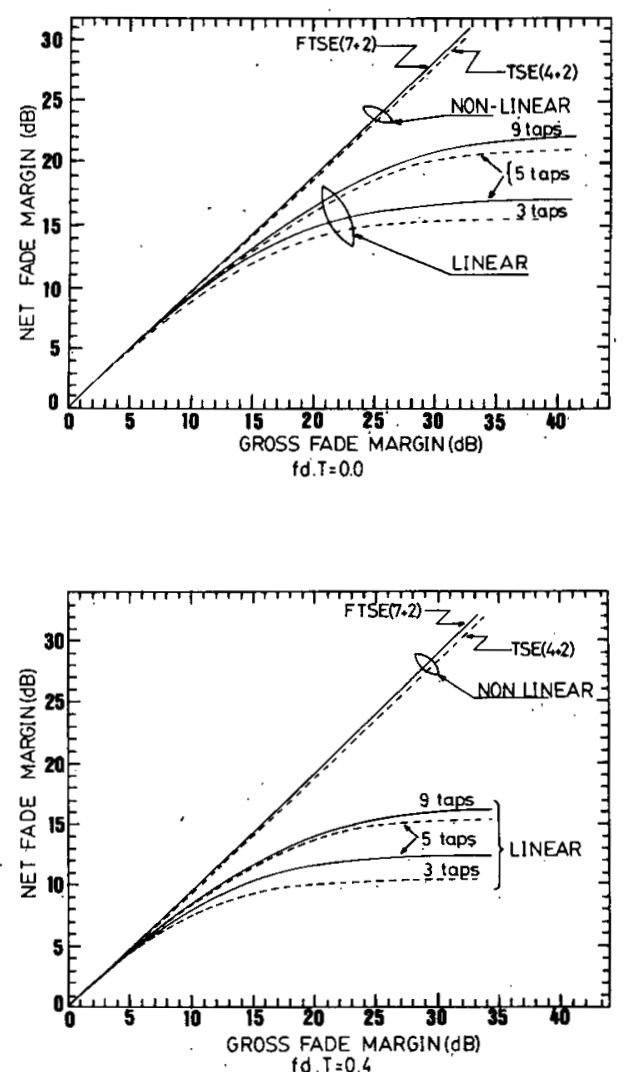

Fig. 3. Net fade margin versus gross fade margin for 16-QAM modulation and minimum-phase fading. Continuous line: FTSE. Dashed line: TSE.

$f_{d} T=0$ and 0.4 . The linear baseband equalizers analyzed have been TSE with three and five taps and FTSE with seven and nine taps. The nonlinear baseband equalizers analyzed were TSE with $N=4$ taps in the linear part and 2 taps in the recursive part, and FTSE with $N=7$ taps in the linear part and 2 taps in the recursive part. Figs. 3-6 show the NFM [see (1)] versus the gross fade margin (GFM) performance for BER $=10^{-3}$ in the presence of minimum-phase fading. The performance of the nonlinear equalizer structures is clearly superior to that of the linear ones. In all the cases analyzed above, the NFM is at most $5 \mathrm{~dB}$ less than the GFM. On the other hand, FTSE performs better than TSE when both compensate for the same number of interfering samples. If we choose the number of taps as a comparison basis, TSE seems to be the best for 16 QAM; however, for the analyzed cases, 32 QAM, $64 \mathrm{QAM}$, and $128 \mathrm{QAM}, f_{d} T=0.4$, and five taps, FTSE performs better. Figs. 7-9 show the NFM versus the GFM performance for $\mathrm{BER}=10^{-3}$ in the presence of nonminimum-phase fading. The reference tap position has been chosen to be $N-1$ [6]. For 16 QAM and 32 QAM, the NFM is at most $10 \mathrm{~dB}$ less than the GFM. However, for 64 QAM and 128 QAM the NFM degradation is quite noticeable. That is due to the fact that the $(N-1)$ th tap value $C_{N-1}$ is dominant for $A<10$ and the $N$ th tap value $C_{N}$ is dominant for $A>20 \mathrm{~dB}$. Then there appears to be an increase of the rms error for $10 \mathrm{~dB}<A<20 \mathrm{~dB}$ that causes an excessive degradation for the high-level 64 QAM and 128 QAM. This fact is explained as follows.
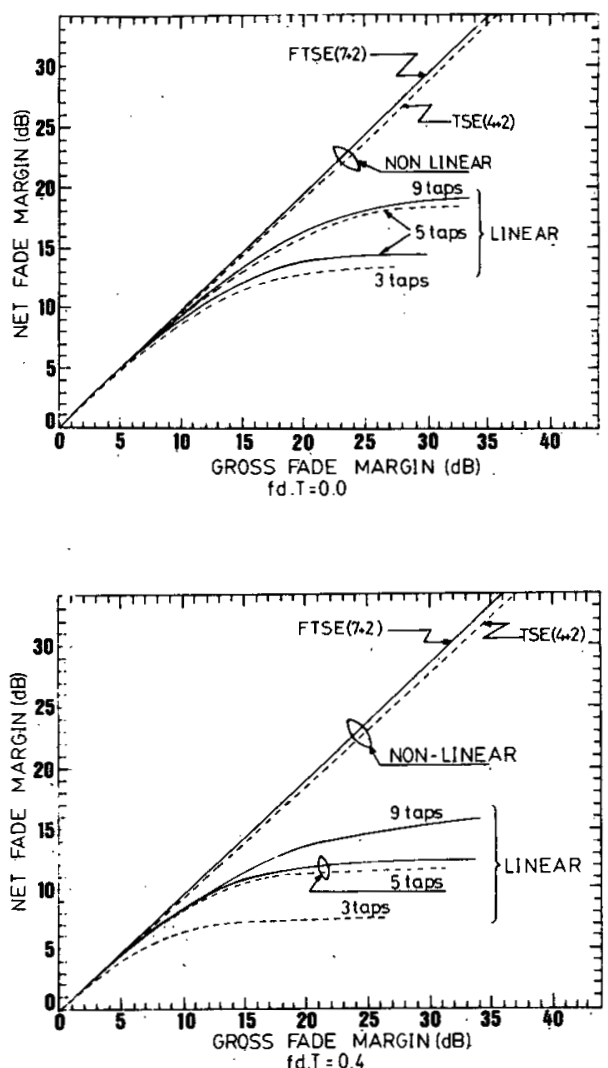

Fig. 4. Net fade margin versus gross fade margin for 32-QAM modulation and minimum-phase fading. Continuous line: FTSE. Dashed line: TSE.
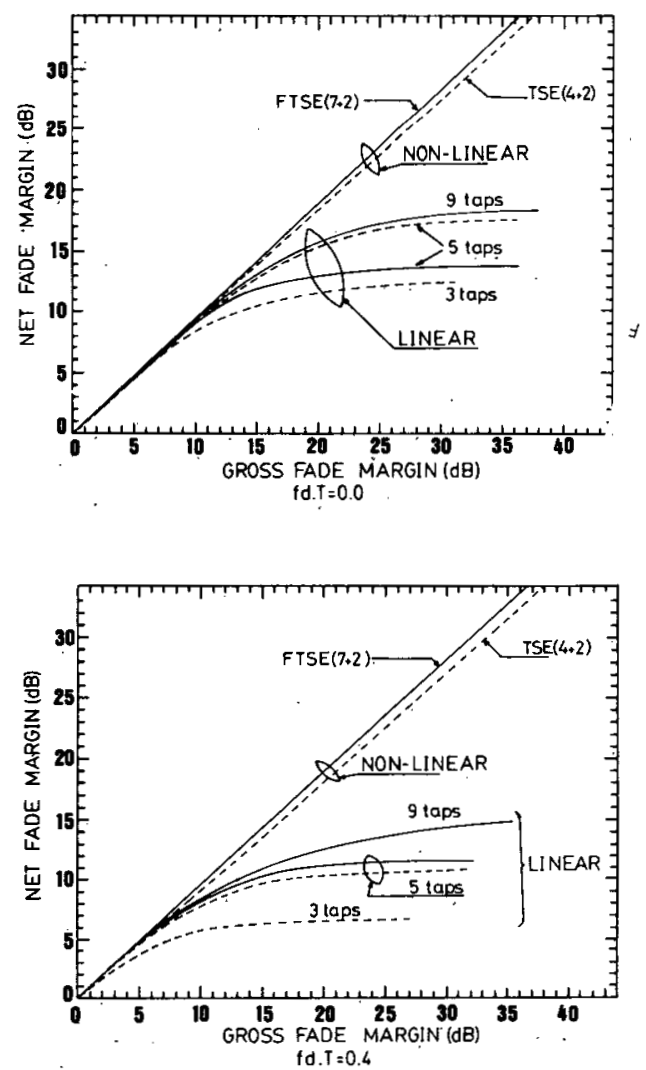

Fig. 5. Net fade margin versus gross fade margin for 64-QAM modulation and minimum-phase fading. Continuous line: FTSE. Dashed line: TSE. 

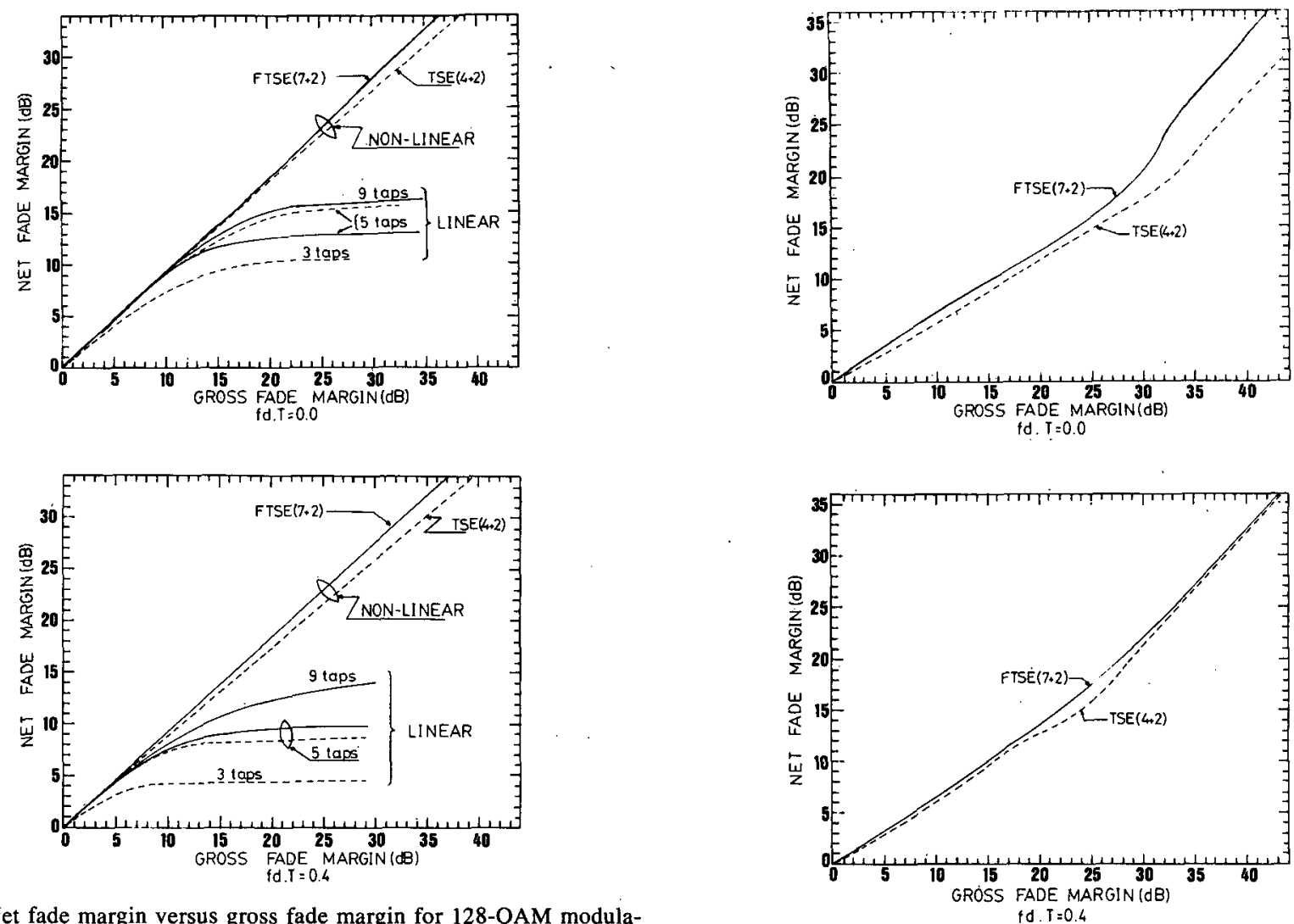

Fig. 6. Net fade margin versus gross fade margin for 128-QAM modulation and minimum-phase fading. Continuous line: FTSE. Dashed line: TSE.
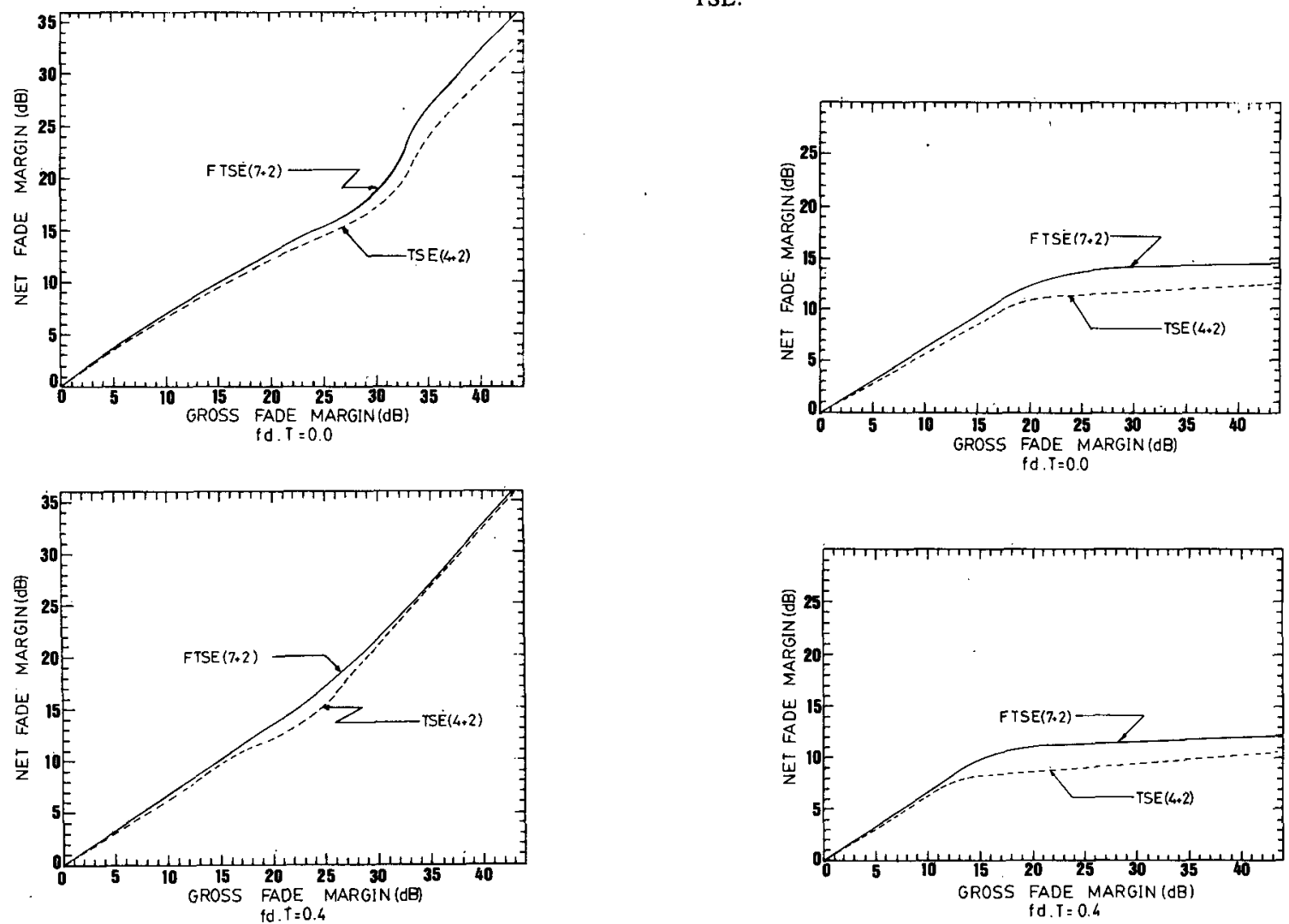

Fig. 7. Net fade margin versus gross fade margin for 16-QAM modulation and nonminimim-phase fading. Continuous line: FTSE. Dashed line: TSE.

Fig. 8. Net fade margin versus gross fade margin for 32-QAM modulation and nonminimum-phase fading. Continuous line: FTSE. Dashed line: TSE.

Fig. 9. Net fade margin versus gross fade margin for 64-QAM modulation and nonminimum-phase fading. Continuous line: FTSE. Dashed line: TSE. 


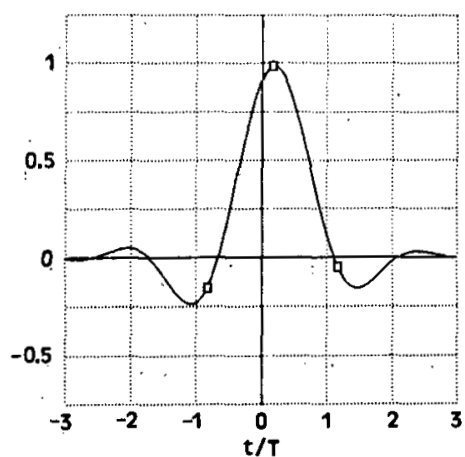

(a)

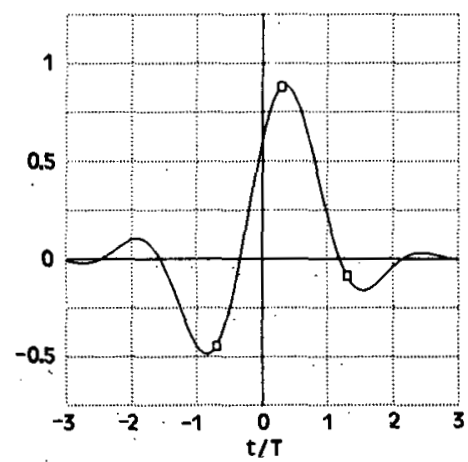

(b)

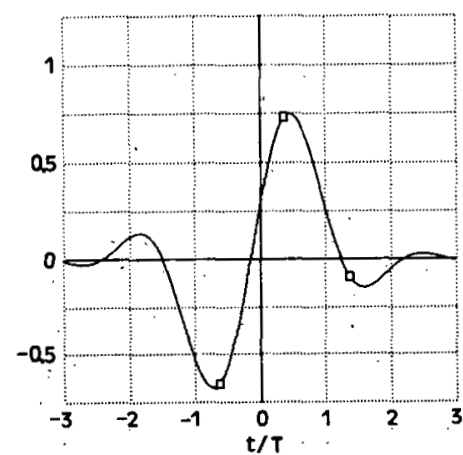

(c)

Fig. 10. Nonequalized impulse responses for nonminimum-phase fading, $f_{d} T=0$, and $\tau / T=0.2205$. The square marks show the most meaningful sample values. (a) Fading depth $=7 \mathrm{~dB}$. (b) Fading depth $=15$ dB. (c) Fading depth $=30 \mathrm{~dB}$

In order to emphasize the relevant aspects of the phenomenon, we considered a centered fade and only the two most important interfering samples in the nonequalized global impulse response $h(t)$. At the input of the $(N-$ 1 th and $N$ th taps we have the signal values

$$
V_{l} \simeq d_{l-1} h_{1}+d_{l} h_{0}+d_{l+1} h_{-1}
$$

and

$$
V_{l-1} \simeq d_{l-2} h_{1}+d_{l-1} h_{0}+d_{l} h_{-1}
$$

respectively. For $A \approx 10 \mathrm{~dB}$ (see Fig. 10(a) for $A=7$ $\mathrm{dB})$, we have $\left|h_{0}\right|>\left|h_{1}\right|$ and $\left|h_{0}\right|>\left|h_{-1}\right|$. Hence, $\vec{C}_{N-1}$ becomes noticeable to enhance the contribution of $V_{l}$, which contains the useful information $d_{l} \dot{h}_{0}$ as its dominant term. Conversely, $C_{N}$ diminishes to reduce the contribution of $V_{l-1}$, whose dominant term $d_{l-1} h_{0}$ is ISI.

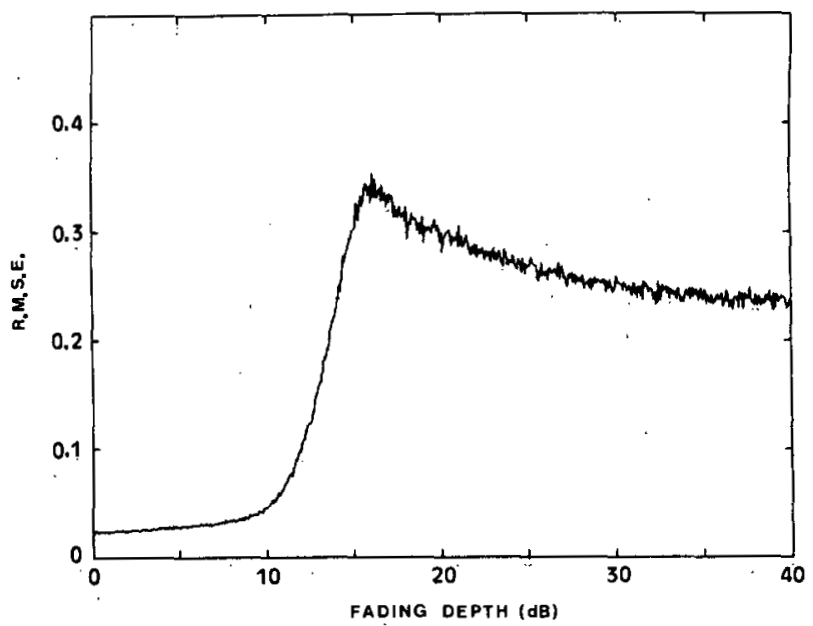

(a)

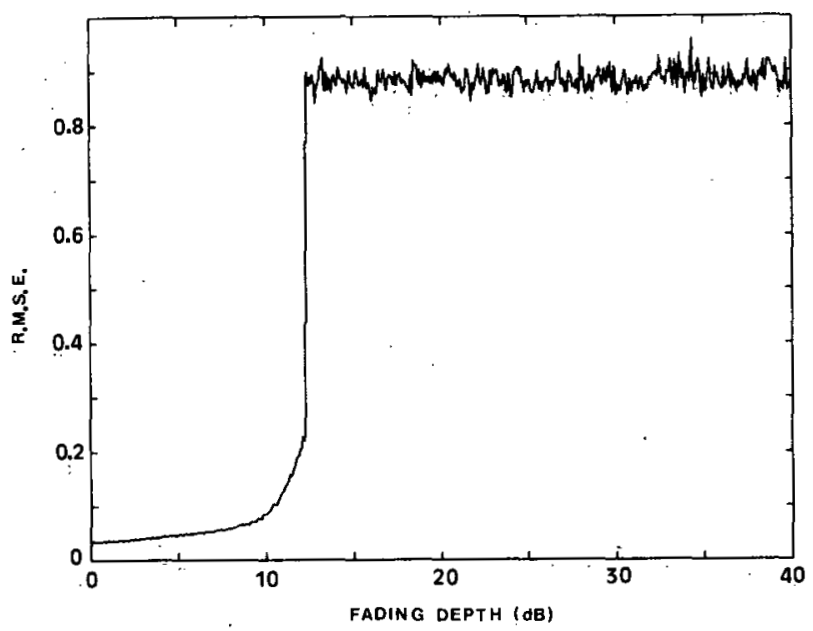

(b)

Fig. 11. Root mean square error against fading depth for nonminimumphase fading. Fading variation rate: $40 \mathrm{~dB} / \mathrm{s}$.(a) 32-QAM modulation (b) 64-QAM modulation.

For $A \subsetneq 20 \mathrm{~dB}$ (see Fig. 10(c) for $A=30 \mathrm{~dB}$ ), $\left|h_{0}\right|$ $=\left|h_{-1}\right|$ and $\left|h_{0}\right| \gg\left|h_{1}\right|$. Now $V_{l}$ has as much useful information $d_{l} h_{0}$ as ISI due to nondetected symbols $d_{l+1} h_{-1}$. On the other hand, $V_{l-1}$ contains useful information due to the precursor sample, $d_{l} h_{-l}$, and ISI due to detected symbols $d_{l-1} h_{0}$. Then the equalizer strategy is to increase $C_{N}$ and to decrease $C_{N-1}$ in order to detect the useful information from $V_{l-1}$ ( $N$ th tap input signal) and to reduce the ISI, due to the nondetected symbols, by means of $C_{N-1}$. The remarkable amount of ISI induced by $d_{l-1} h_{0}$ will be cancelled through the recursive part of the equalizer. For $10 \mathrm{~dB} \approx A \approx 20 \mathrm{~dB}$ in the equalizer behavior, an intermediate situation characterized by an increase and a posterior decrease of the rms error value appears. This fact has been confirmed by a computed simulation that took into account a dynamic fading activity characterized by a fading variation rate of $40 \mathrm{~dB} / \mathrm{s}$. Fig. 11 shows the rms error obtained for 32-QAM and 64 QAM systems. In the last case, it can be noted how the dramatic rms error increase beyond approximately 12.5 $\mathrm{dB}$ impedes the equalizer from working properly. The rms 
error increase for $10 \mathrm{~dB} \approx A \approx 20 \mathrm{~dB}$ would also explain the poorer NFM performance in this range, as shown in Figs. 7 and 8.

\section{CONCLUSION}

A comparative study of 16-QAM, 32-QAM, 64-QAM, and 128-QAM $140 \mathrm{Mbit} / \mathrm{s}$ digital radio in the presence of multipath fading has been carried out. Nonminimumphase and minimum-phase fading and receiver structures including TSE and FTSE have been considered.

In order to use the BER as a performance parameter, new upper and lower bounds on BER in dispersive digital radio have been developed. The obtained results, shown as NFM versus GFM curves, allow comparison of the equalizer performance of the different considered structures. In particular, nonlinear equalizers perform better than linear equalizers for the minimum-phase fading cases. However, that is not true for nonminimum-phase fading cases where the performance limitation of the nonlinear equalizers is noticeable for high-level 64 QAM and 128 QAM. On the other hand, linear TSE seems to perform better than linear FTSE for centered fading, but for noncentered fading $\left(f_{d} T=0.4\right)$ and 32 QAM, 64 QAM, and $128 \mathrm{QAM}$ the converse is true.

\section{REFERENCES}

[1] M. Borgne, "Comparison of high-level modulation schemes for highcapacity digital radio systems," IEEE Trans. Commun., vol. COM33, pp. 442-449, May 1985.

[2] P. Dupuis, M. Joindot, A. Leclert, and D. Soufflet, "16-QAM modulation for high capacity digital radio system," IEEE Trans. Commun., vol. COM-27, pp. 1771-1782, Dec. 1979.

[3] A. Milewski, "New simple and efficient bounds on the probability of error in the presence of intersymbol and Gaussian noise," IEEE Trans. Commun., vol. COM-25, pp. 1218-1222, Oct. 1977.

[4] W. D. Rummler, "A new selective fading model: Application to propagation data," Bell Syst. Tech. J., vol. 58, pp. 1037-1071, May-June 1979.

[5] R. Agustí and F. Casadevall, "Pseudoerror monitor for 16QAM 140 Mbit/s digital radio," IEEE Trans. Commun., vol. COM-34, pp. 606613, June 1986

[6] A. Leclert and P. Vandamme, "Decision feedback equalization of dispersive radio channels," IEEE Trans. Commun., vol. COM-33, pp. 676-684, July 1985 .

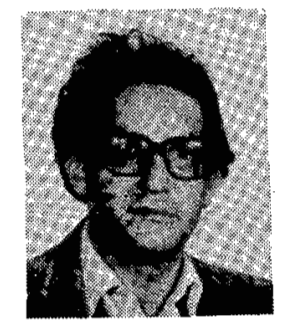

Ramón Agustí (M'78) was born in Riba-roja d'Ebre, Spain, on August, 15, 1951. He received the Engineer of Telecommunications degree from de Universidad Politécnica de Madrid, Spain, in 1973 and the Ph.D. degree from the Universidad Politécnica de Catalunya, Spain, in 1978.

In 1973 he joined the Escuela Técnica Superior de Ingenieros de Telecomunicación de Barcelona, Spain, where he has been an Associate Professor since 1978. He has been working in the field of digital communications, with particular emphasis on digital radio and its performance under multipath propagation conditions. In the last few years he has also been concerned with the performance analysis and development of frequency-hopped spread-spectrum systems.

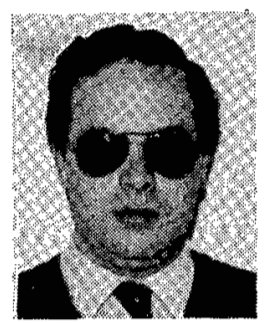

Fernando Casadevall was born in Barcelona, Spain, on April 22, 1955. He received the Engineer of Telecommunication and Ph.D. degrees from the Universidad Politécnica de Catalunya, Spain, in 1977 and 1983 , respectively.

In 1978 he joined the Escuela Técnica Superior de Ingenieros de Telecomunicación de Barcelona, Spain, where he has been as Associate Professor since 1983. After graduation he was concerned with equalization techniques for digital fiber optic systems. His present research interests are in the areas of digital radio and spread-spectrum mobile communications.

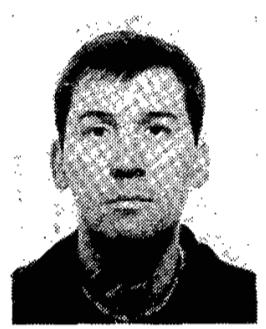

Juan J. Olmos was born in Palma de Mallorca, Spain, on December 6, 1956. He received the Engineer of Telecommunications degree from the Universidad Politécnica de Catalunya, Spain, in 1983.

In 1983 he joined the Escuela Técnica Superior de Ingenieros de Telecomunicación de Barcelona, Spain, where he is currently pursuing the Ph.D. degree in the area of digital radio. 